\title{
The Rise of Tunnel Endoscopic Surgery: A Case Report and Literature Review
}

\author{
Mingyan Cai,, ${ }^{1}$ Jinzhong Chen, ${ }^{2}$ Pinghong Zhou, ${ }^{1}$ and Liqing Yao ${ }^{1}$ \\ ${ }^{1}$ Endoscopy Center and Endoscopy Research Institute, Zhongshan Hospital, Fudan University, 180 Fenglin Road, \\ Shanghai 200032, China \\ ${ }^{2}$ Department of Gastrointestinal Endoscopy, No.1 Hospital Affiliated to Xiamen University, Fujian, Xiamen 361003, China
}

Correspondence should be addressed to Pinghong Zhou, zhou1968@yahoo.cn

Received 30 April 2012; Accepted 5 August 2012

Academic Editors: S. Kikuchi, R. Kochhar, G. Kouraklis, I. D. Kyriazanos, R. J. L. F. Loffeld, and J. Wig

Copyright () 2012 Mingyan Cai et al. This is an open access article distributed under the Creative Commons Attribution License, which permits unrestricted use, distribution, and reproduction in any medium, provided the original work is properly cited.

There has been booming interest in natural orifice transluminal surgery since it was first described. Several techniques first developed for the safe transluminal access now derive into independent endoscopic surgical procedures. In this paper, we describe a case treated by a novel procedure by submucosal tunnelling technique and provide a literature review of the rise of tunnel endoscopic surgery.

\section{Introduction}

Tunnel endoscopy is a novel approach which was initially developed for the purpose of establishing an access for natural orifice transluminal endoscopic surgery (NOTES) [1-3]. Meanwhile, it brought a great idea for endoscopic procedures by using the submucosal tunnel as an operating space. The emerge of peroral endoscopic myotomy (POEM) for treating esophageal achalasia marked the rising of a new branch of therapeutic endoscopy [4]. Our group defines it as tunnel endoscopic surgery which includes several novel procedures utilizing a submucosal tunnel as an operating space. We innovate a new method by tunnelling technique for treating submucosal tumors originating from muscularis propria [5]. Herein we report a case operated by this novel method: submucosal tunnelling endoscopic resection (STER).

\section{Case Report}

A 55-year-old male patient presented with a chief complaint of discomfort behind his sternum when swallowing. A submucosal tumor about $1.5 \mathrm{~cm} \times 2.0 \mathrm{~cm}$ was found at middle esophagus ( $26 \mathrm{~cm}$ from the incisors) by gastroscopy. The endoscopic ultrasound revealed the tumor originated in the muscularis propria. After obtaining the institutional review board approval and informed consent, STER was arranged for this patient and was performed by an experienced endoscopist (PHZ).

The procedure was carried out under general anaesthesia with trachea intubation. It was consisted of the following steps: mucosal injection and incision, submucosal tunnelling, tumor resection, and mucosal closure (Figure 1). The equipment needed was the same as that of standard endoscopic submucosal resection (ESD) [6-8]. The mucosal injection and incision point were chosen at approximately $5 \mathrm{~cm}$ above the lesion site. After mucosal injection, a $2 \mathrm{~cm}$ mucosal incision was made by a hook knife. Then, a submucosal tunnel was created from the incision site to $1-2 \mathrm{~cm}$ below the lesion as long as it was fully exposed by ESD technique. The tumor was completely dissected from the muscularis layer while the adventitia being intact. After the specimen retrieval and the hemostasis in the submucosal tunnel, the mucosal incision site was close by metallic clips. The whole procedure took 30 minutes.

The patient felt no pain after procedure though a subcutaneous emphysema and a minor left pneumothorax were observed on CT scan on postoperative day 1. No further intervention was necessary. The patient was given full liquid diet on postoperative day 2. The symptom of dysphagia relieved immediately. He was discharged uneventfully. The 


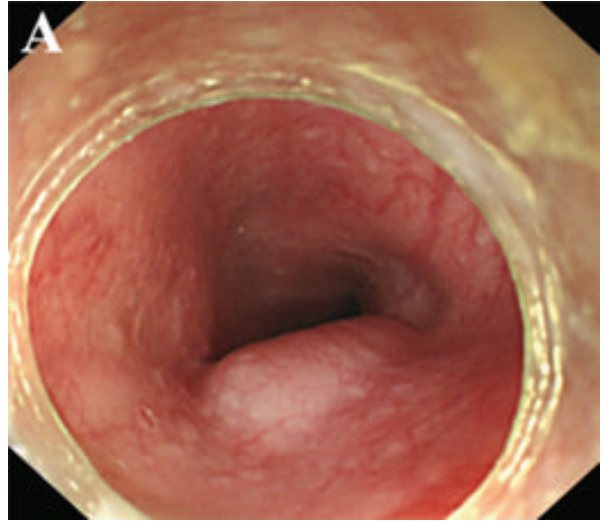

(a)

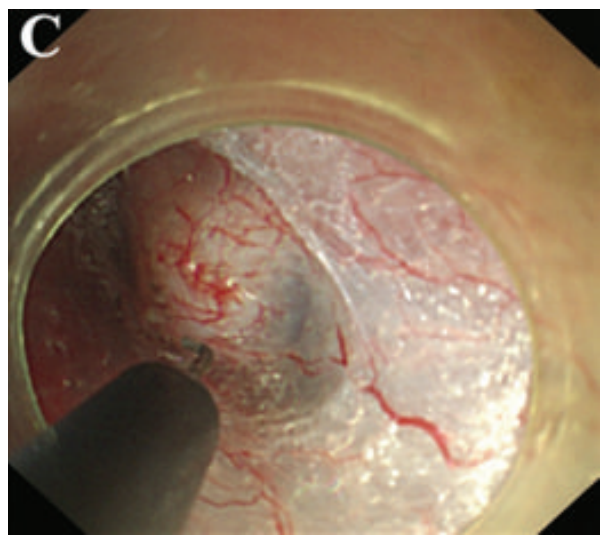

(c)



(e)

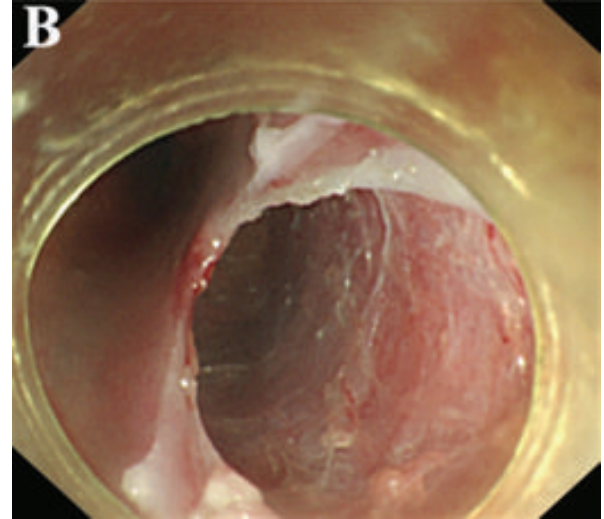

(b)

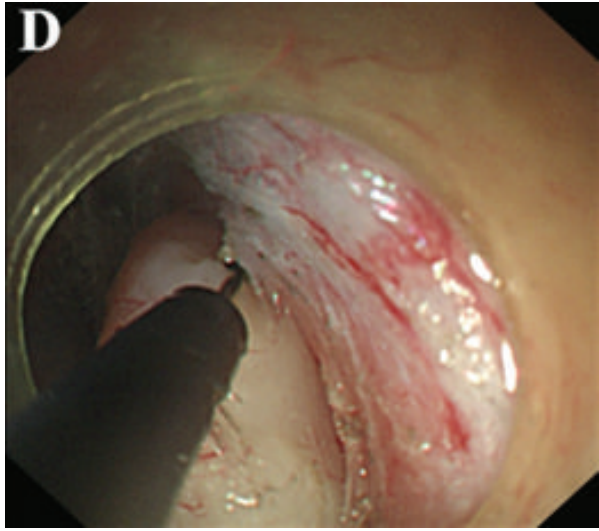

(d)

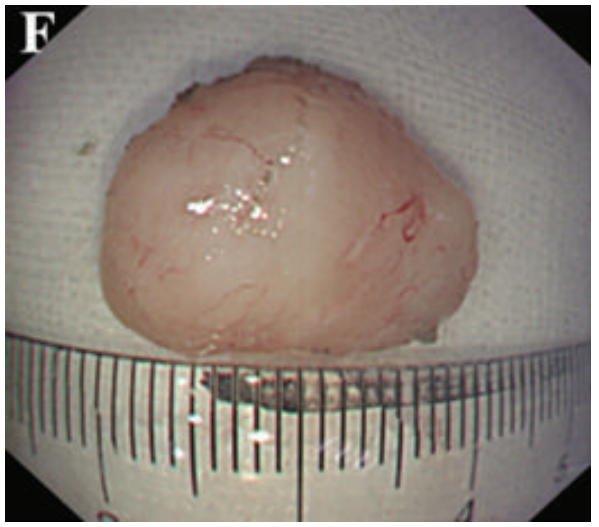

(f)

Figure 1: The endoscopic tunnelling submucosal resection procedure. (a) A submucosal tumor was found in esophagus (26 cm from incisors). (b) After mucosal injection, a submucosal tunnel (about $5 \mathrm{~cm}$ long) was created by ESD technique. (c) The tumor was successfully exposed in the tunnel. (d) Tumor resection was being carried out in the tunnel. (e) The mucosal incision site was sealed by hemostatic clips.

(f) The resected specimen was $1.5 \mathrm{~cm} \times 2.0 \mathrm{~cm}$.

pathology result showed an esophageal leiomyoma with clear margins (Figure 2). The follow-up esophagoscopy revealed a well-healed mucosa (Figure 3).

\section{Discussion and Literature Review}

This case demonstrates that the application of tunnelling technique is feasible for the en bloc resection of upper gastrointestinal submucosal tumors from deep layer. Our preliminary data of large scales is in publication [5]. Herein we review the development and the new emerging procedures of tunnel endoscopic surgery.

\section{Tunnel Endoscopic Surgery in Animal Model}

A blossomed interest has been aroused in NOTES since it was first reported in 2004 [9]. Tunnel endoscopy, also referred as "submucosal endoscopy with mucosal flap safety valve" 


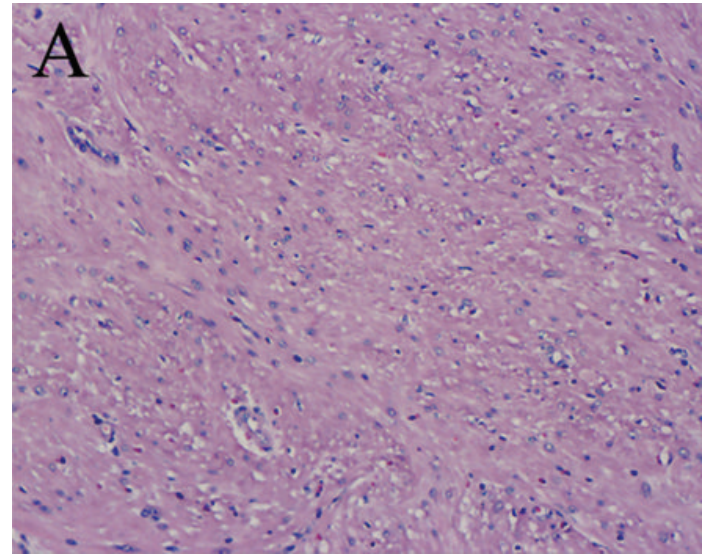

(a)

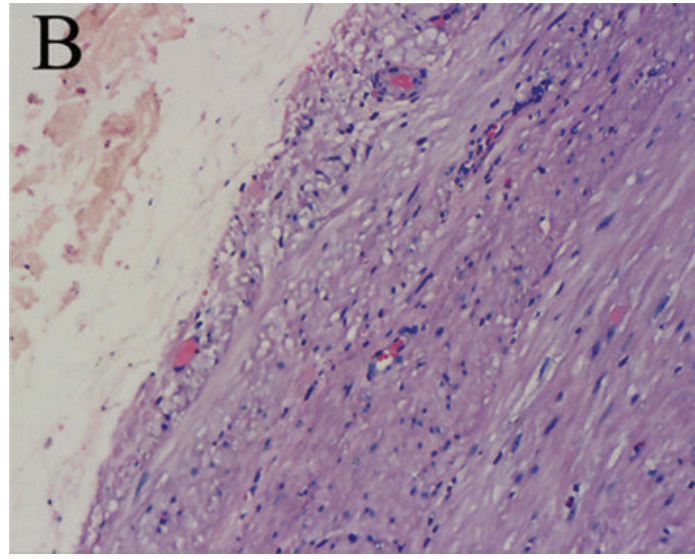

(b)

Figure 2: Pathology of the resected specimen. The pathology showed a leiomyoma (a) and clear margins (b) (magnifying $\times 200)$.

(SEMF) technique $[2,10-12]$ or "self-approximating transluminal access technique" (STAT) $[1,13]$, was developed for the safe access of NOTES in porcine model in 2007. The two independent groups shared the similar thoughts by creating an ST from the wall of natural orifice; however, the methodology of tunnelling technique they used varied a little from each other. In SEMF technique, the authors created submucosal tunnel by high-pressure carbon dioxide $\left(\mathrm{CO}_{2}\right)$ injection and balloon dissection $[2,3]$. The shortcoming of this method is that high-pressure $\mathrm{CO}_{2}$ injection and balloon dissection may create an excessive large submucosal tunnel that partial necrosis of the overlying free mucosa occurred in three of four pigs [3]. Besides, a fluid injection was still needed for the prevention of gas escape. While in STAT, a fluid cushion of normal saline was made, and submucosal tunnel was created by the sharp and blunt dissection of a grasping forcep and the leading edge of the endoscopy itself [1]. The evidence to date suggests that STAT allows safe and reliable translumenal access and the removal of small-to-moderate-sized $(<8 \times 3 \mathrm{~cm})$ specimens during NOTES procedures in animal models [14]. A comparative study of four different transgastric access showed that an extended submucosal tunnel yielded the best leak resistance, which is superior to standard transgastric access methods and rival handsewn interrupted stitches [15]. However, neither balloon dissection nor forcep dissection could avoid the risk of bleeding when tunnelling. The application of endoscopic submucosal dissection (ESD) technique in tunnel endoscopy optimized this transluminal access [16].

Actually, the attempt of tunnel endoscopic surgery also dated back to 2007. That means tunnel endoscopy was not only developed as an access for NOTES but also considered as an intervention treatment. Submucosal endoscopic esophageal myotomy was depicted as a novel experimental approach for the treatment of achalasia. A submucosal saline lift was created approximately $5 \mathrm{~cm}$ above the lower esophageal sphincter (LES), and a small nick was made in the mucosa in order to facilitate the introduction of a dilating balloon. After dilation, the scope was introduced over the balloon into the submucosal space and advanced toward the now visible fibers of the LES. The circular layer of muscle was then cleanly incised using an electrocautery knife in a distalto-proximal fashion, without complications [17].

\section{Tunnel Endoscopic Surgery in Human}

The milestone of tunnel endoscopic surgery in clinical application is POEM for treating esophageal achalasia. After submucosal injection, the endoscopy enters the submucosal space and begins submucosal tunnelling. A long submucosal tunnel is created to $3 \mathrm{~cm}$ distal to the gastroesophageal junction (GEJ). Then, endoscopic myotomy is carried out from $3 \mathrm{~cm}$ below the mucosal incision point in a proximal to distal manner to a total length of $10 \mathrm{~cm}$. Finally the mucosal incision site is closed by hemostatic clips [4]. In all 17 cases including 5 difficult ones (sigmoid type), the patients had a great improvement in clinical manifestations and manometric findings. No procedure related serious complication encountered. The success of the procedure is the very first attempt for applying tunnel endoscopic surgery for treating human diseases. Moreover, utilizing the submucosal space not only as a transluminal access but also as an independent surgical method marks the beginning of tunnel endoscopic surgery as a new branch of interventional endoscopy.

Inspired by the success in POEM and other tunnel endoscopic applications in NOTES, we innovated the novel procedure based on submucosal tunnelling technique. In the reported case, STER was carried out by creating a submucosal tunnel from $5 \mathrm{~cm}$ distal to $1-2 \mathrm{~cm}$ proximal to the submucosal tumor by a hook knife to provide enough operation space for the next step: endoscopic dissection. The tumor was dissected carefully by an insulated-tipped knife from the muscularis propria layer, keeping the adventitia intact as possible. If a perforation occurs inevitably, the overlying mucosa can provide a seal for the wall defect. Finally, the mucosal entry point was closed with hemostatic clips. It is a promising procedure for several reasons. (a) For submucosal tumors originating from deeper layers of the gut wall, perforation is no longer a fearful issue during the procedure. Perforation is only a procedure-related event and can be successfully 


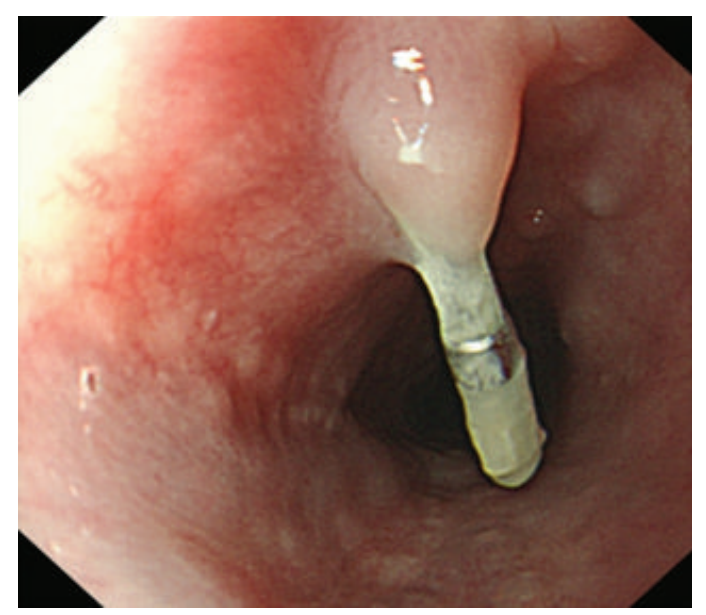

(a)

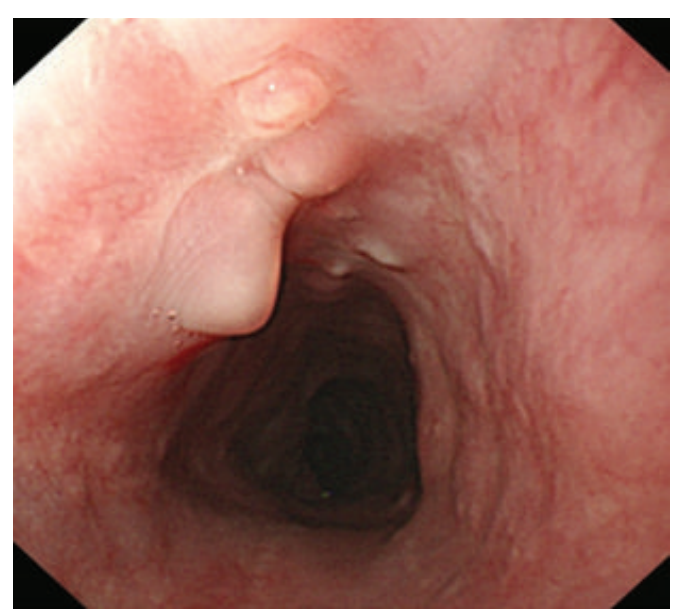

(b)

Figure 3: Follow-up esophagoscopy. On postoperative month 2, the follow-up esophagoscopy demonstrated a well-healed mucosa.

managed after tumor resection. After the withdrawal of endoscopy from the submucosal tunnel, the overlying free mucosa serves as a sealant flap over the myomectomy site and immediately holds the air escaping into the mediastinum or abdomen even in an unclipped ex vivo model [1]. Thus, it provides abundant time for mucosa closure. (b) An extended submucosal tunnel of $5 \mathrm{~cm}$ showed the best leak resistance compared with standard transgastric access methods such as linear incision and balloon dilation [15]. (c) The equipment used in STER is the same as that of ESD, while ESD is an established method for treating early mucosal carcinoma or submucosal tumors.

\section{Conclusion}

The development of tunnel endoscopy and its variation of surgical procedures are inspiring for both gastroenterologists and surgeons. We believe that the future of flexible endoscopy is not only operating in the natural orifice but also within and outside the gastrointestinal wall. The latter needs more innovation and courage for the endoscopists.

\section{Author's Contributions}

M. Cai and J. Chen contributed equally to this work; M. Cai, J. Chen, and P. Zhou are responsible for conception, and design, data acquisition and analysis, and manuscript writing. P. Zhou performed the procedure. P. Zhou and L. Yao are responsible for financial support, manuscript revision, and final approval of the manuscript.

\section{Conflict of Interests}

The authors declare that they have no conflict of interests.

\section{Acknowledgment}

This work is supported by Medical Leading Project of Shanghai Municipal Science and Technology Committee (10411969600).

\section{References}

[1] M. T. Moyer, E. M. Pauli, R. S. Haluck, and A. Mathew, "A selfapproximating transluminal access technique for potential use in NOTES: an ex vivo porcine model (with video)A figure is presented," Gastrointestinal Endoscopy, vol. 66, no. 5, pp. 974978, 2007.

[2] K. Sumiyama, C. J. Gostout, E. Rajan, T. A. Bakken, and M. A. Knipschield, "Transesophageal mediastinoscopy by submucosal endoscopy with mucosal flap safety valve technique," Gastrointestinal Endoscopy, vol. 65, no. 4, pp. 679-683, 2007.

[3] K. Sumiyama, C. J. Gostout, E. Rajan, T. A. Bakken, M. A. Knipschield, and R. J. Marler, "Submucosal endoscopy with mucosal flap safety valve," Gastrointestinal Endoscopy, vol. 65, no. 4, pp. 688-694, 2007.

[4] H. Inoue, H. Minami, Y. Kobayashi et al., "Peroral endoscopic myotomy (POEM) for esophageal achalasia," Endoscopy, vol. 42, no. 4, pp. 265-271, 2010.

[5] M. D. Xu, M. Y. Cai, P. H. Zhou et al., "Submucosal tunneling endoscopic resection: a new technique for treating upper GI submucosal tumors originating from the muscularis propria layer (with videos)," Gastrointestinal Endoscopy, vol. 75, pp. 195-199, 2011.

[6] M. Kato, "Endoscopic submucosal dissection (ESD) is being accepted as a new procedure of endoscopic treatment of early gastric cancer," Internal Medicine, vol. 44, no. 2, pp. 85-86, 2005.

[7] T. Oyama, A. Tomori, K. Hotta et al., "Endoscopic submucosal dissection of early esophageal cancer," Clinical Gastroenterology and Hepatology, vol. 3, no. 7, supplement 1, pp. S67-S70, 2005.

[8] T. Gotoda, H. Yamamoto, and R. M. Soetikno, "Endoscopic submucosal dissection of early gastric cancer," Journal of Gastroenterology, vol. 41, no. 10, pp. 929-942, 2006.

[9] A. N. Kalloo, V. K. Singh, S. B. Jagannath et al., "Flexible transgastric peritoneoscopy: a novel approach to diagnostic and therapeutic interventions in the peritoneal cavity," Gastrointestinal Endoscopy, vol. 60, no. 1, pp. 114-117, 2004.

[10] K. Sumiyama, C. J. Gostout, E. Rajan et al., "Transgastric cholecystectomy: transgastric accessibility to the gallbladder improved with the SEMF method and a novel multibending therapeutic endoscope," Gastrointestinal Endoscopy, vol. 65, no. 7, pp. 1028-1034, 2007. 
[11] K. Sumiyama, C. J. Gostout, E. Rajan et al., "Pilot study of transesophageal endoscopic epicardial coagulation by submucosal endoscopy with the mucosal flap safety valve technique (with videos)," Gastrointestinal Endoscopy, vol. 67, no. 3, pp. 497-501, 2008.

[12] K. Sumiyama, H. Tajiri, and C. J. Gostout, "Submucosal endoscopy with mucosal flap safety valve (SEMF) technique: a safe access method into the peritoneal cavity and mediastinum," Minimally Invasive Therapy and Allied Technologies, vol. 17, no. 6, pp. 365-369, 2008.

[13] E. M. Pauli, M. T. Moyer, R. S. Haluck, and A. Mathew, "Self-approximating transluminal access technique for natural orifice transluminal endoscopic surgery: a porcine survival study (with video)," Gastrointestinal Endoscopy, vol. 67, no. 4, pp. 690-697, 2008.

[14] M. T. Moyer, E. M. Pauli, J. Gopal, A. Mathew, and R. S. Haluck, "Durability of the self-approximating translumenal access technique (STAT) for potential use in natural orifice translumenal surgery (NOTES)," Surgical Endoscopy and Other Interventional Techniques, vol. 25, no. 1, pp. 315-322, 2011.

[15] S. von Delius, S. Gillen, E. Doundoulakis et al., "Comparison of transgastric access techniques for natural orifice transluminal endoscopic surgery," Gastrointestinal Endoscopy, vol. 68, no. 5, pp. 940-947, 2008.

[16] F. Yoshizumi, K. Yasuda, K. Kawaguchi, K. Suzuki, N. Shiraishi, and S. Kitano, "Submucosal tunneling using endoscopic submucosal dissection for peritoneal access and closure in natural orifice transluminal endoscopic surgery: a porcine survival study," Endoscopy, vol. 41, no. 8, pp. 707-711, 2009.

[17] P. J. Pasricha, R. Hawarl, I. Ahmed et al., "Submucosal endoscopic esophageal myotomy: a novel experimental approach for the treatment of achalasia," Endoscopy, vol. 39, no. 9, pp. 761-764, 2007. 


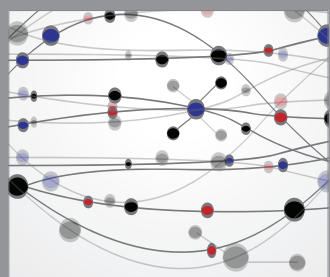

The Scientific World Journal
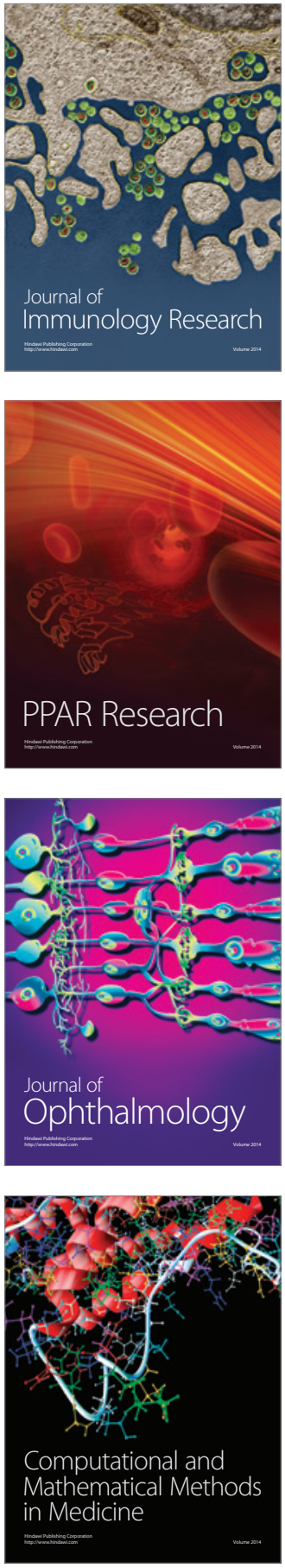

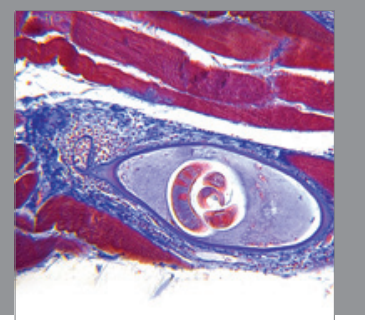

Gastroenterology

Research and Practice
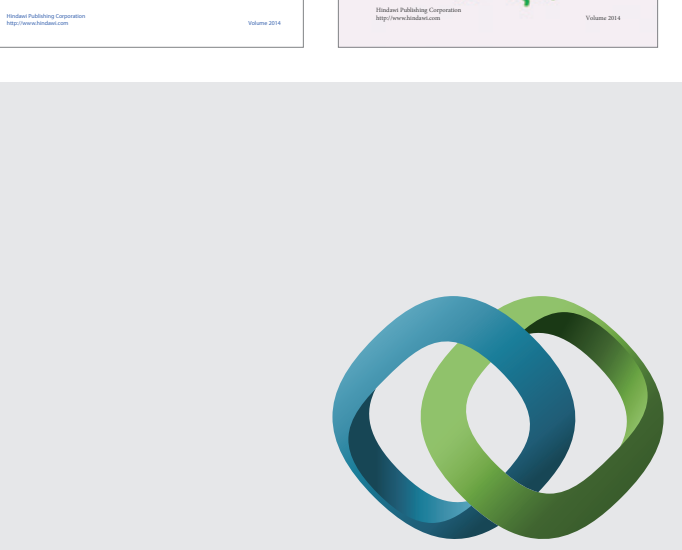

\section{Hindawi}

Submit your manuscripts at

http://www.hindawi.com
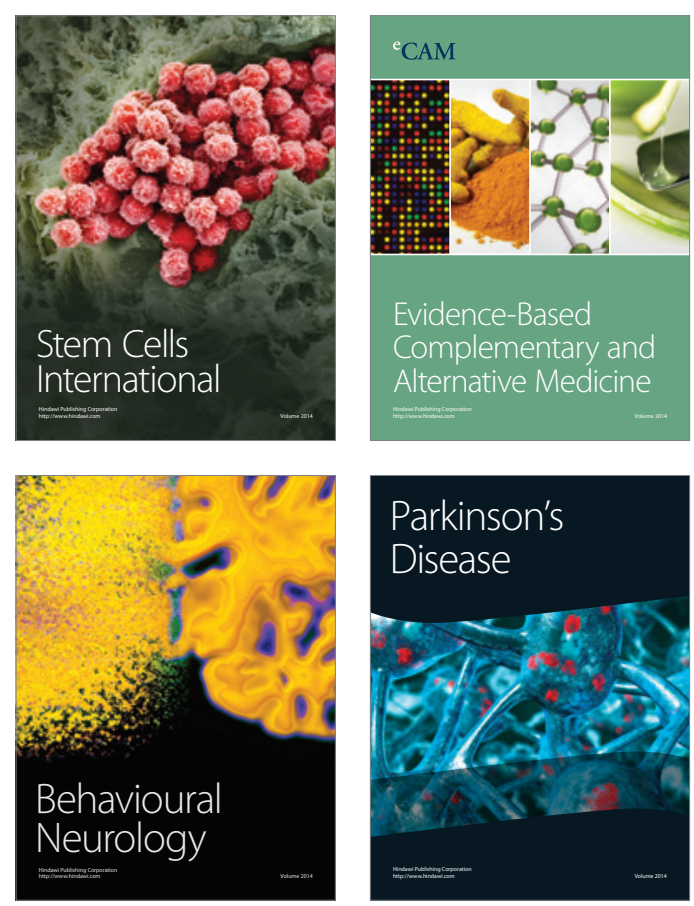

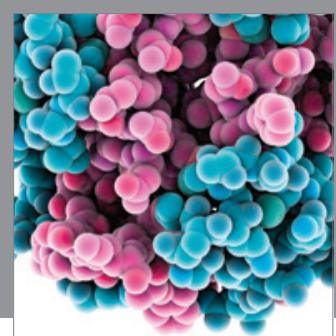

Journal of
Diabetes Research

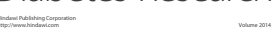

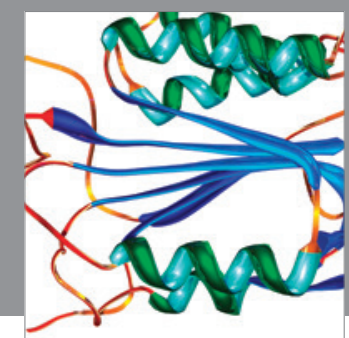

Disease Markers
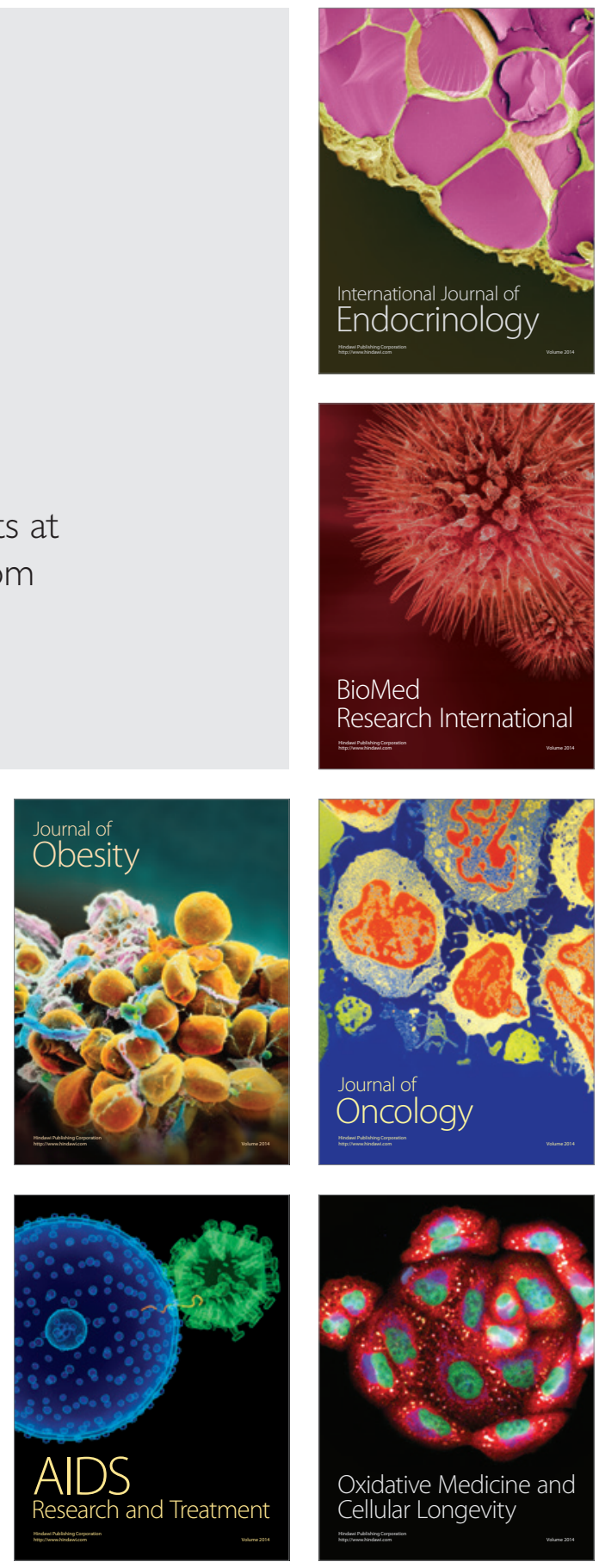